\title{
Elastic constants and homogenized moduli of gypsum structure based on Density Functional Theory
}

\author{
Jia Fu ${ }^{1,2}$ a , Weihui Lin ${ }^{3}$ b \\ ${ }^{1}$ Material science and technology, Xi'an Shiyou University, Xi 'an, 710065, China \\ ${ }^{2}$ MSE department, Southern University of Science and Technology, ShenZhen, 518055, China \\ ${ }^{3}$ School of aerospace, Xi'an Jiaotong University, Xi 'an, 710049, China \\ a* fujia@xsyu.edu.cn, ${ }^{b}$ linweihui@stu.xjtu.edu.cn
}

\begin{abstract}
Keywords: DFT calculation;Nanoscale modeling;Gypsum crystal;Elastic constants;Homogenized moduli.

Abstract. Elastic constants and homogenized properties of gypsum structure were investigated by first-principles method. The gypsum (chemical formula of $\mathrm{CaSO}_{4} \cdot 2 \mathrm{H}_{2} \mathrm{O}$ ) is an evaporite mineral and a kind of hydration product of anhydrite (chemical formula: $\mathrm{CaSO}_{4}$ ). Elastic constants are calculated based on Density Functional Theory (DFT), which can also contribute to provide information for investigate the anisotropy and mechanical properties of gypsum polycrystals. In addition, based on elastic constants (13 independent constants) of the monoclinic crystal, elastic properties of gypsum polycrystals are obtained. The Young's modulus, shear modulus, bulk modulus and Poisson's ration are derived. Therefore, it is fairly meaningful to study the elastic constants to understand the physical, chemical and mechanical properties of gypsum structure. Elastic constants can be used as the measure criterion of the resistance of a crystal to an externally applied stress. The calculated parameters are in excellent agreement with reference by Huang et al..
\end{abstract}

\section{Introduction}

As its appreciable advantages such as load-bearing capacity, durability, maneuverability, flowability..., the building materials and their properties have been deeply investigated in the dedicated scientific literatures ${ }^{[1,2,3]}$. DFT as a first-principles theory and a solid band theory in quantum mechanics has own a great success in linking physical properties and molecular structure, the calculation with exact accuracy but for low computational efficiency for macromolecular structure $^{[4]}$. Moreover, the cement-based materials were separately investigated and multi-body (many-body calculation) structures were calculated on the basis of certain modelling and simulations ${ }^{[5,6,7,8]}$.

Polycrystalline structure constituted by a single crystal structure contains a variety of information (eg. orientation) and the properties of a single crystal, such as anisotropy. Molecular pressure characteristics are critical to better understand the mechanical properties of the materials at the atomic scale ${ }^{[9]}$. Within the mechanics of crystal structures, the homogenization and localization can be estimated ${ }^{[10]}$. The Reuss-Voigt-Hill estimation method is used to determine homogenized moduli of the polycrystalline material composed of a single crystal, eg. the certain stress of Reuss model ${ }^{[11]}$, the certain strain of Voigt model ${ }^{[12]}$, X-Rays diffraction measurement ${ }^{[13]}$, the Y-parameter ${ }^{[14]}$ and diffraction stress factor ${ }^{[9]}$.

The chemical formula of gypsum is $\mathrm{CaSO}_{4} \cdot 2 \mathrm{H}_{2} \mathrm{O}$, which is an evaporite mineral and a kind of hydration product of anhydrite (chemical formula: $\mathrm{CaSO}_{4}$ ). There is no scientific report about its homogenized moduli. Since the Young's modulus parameter of gypsum is important to the multi-scale model ${ }^{[15]}$, elastic constants of the gypsum crystal are investigated. DFT method can be used to calculate elastic constants of anisotropic gypsum crystals. The crystal is monoclinic, with 13 independent constants. For the homogenization of elastic deformation, especially for polycrystalline structures, the traditional Reuss-Voigt-Hill method is used to calculate elastic moduli of gypsum polycrystals structures. Based on the density functional theory, we focus on the monoclinic gypsum crystal to estimate their homogenized elastic moduli. 


\section{Homogenization of monoclinic polycrystals by RVH estimation}

For an orthotropic monoclinic crystal, stress-strain relation can be defined by the independent elastic stiffness parameters ${ }^{[16]}$ :

$$
\left[\begin{array}{l}
\sigma_{11} \\
\sigma_{22} \\
\sigma_{33} \\
\sigma_{12} \\
\sigma_{13} \\
\sigma_{23}
\end{array}\right]=\left[\begin{array}{cccccc}
c_{11} & c_{12} & c_{13} & 0 & c_{15} & 0 \\
c_{12} & c_{22} & c_{23} & 0 & c_{25} & 0 \\
c_{13} & c_{23} & c_{33} & 0 & c_{35} & 0 \\
0 & 0 & 0 & c_{44} & 0 & c_{46} \\
c_{15} & c_{25} & c_{35} & 0 & c_{55} & 0 \\
0 & 0 & 0 & c_{46} & 0 & c_{66}
\end{array}\right]\left[\begin{array}{l}
\varepsilon_{11} \\
\varepsilon_{22} \\
\varepsilon_{33} \\
\gamma_{12} \\
\gamma_{13} \\
\gamma_{23}
\end{array}\right]
$$

Where, $\sigma$ represents the normal stress and shear stress in each direction (unit: $n N / n m^{2}$ ); $\varepsilon$ and $\gamma$ are the normal strain and shear strain in each direction.

According to the theory of elasticity, under the isothermal strain, the elastic modulus of Helmholtz free energy can be described by the form of the Taylor expansion, of which the coefficients of the polynomial is the elastic coefficient:

$$
\rho_{0} F\left(\eta_{i j}, T\right)=\rho_{0} F\left(\eta_{i j}, T\right)+\frac{1}{2} \sum_{i j k l} c_{i j k l}^{T} \eta_{i j} \eta_{k l}+\ldots+\frac{1}{n !} \sum_{i j k l . .} c_{i j k l . \ldots}^{T} \eta_{i j} \eta_{k l} \ldots
$$

Where, $\eta_{i j}, \eta_{k l}$ and $\eta_{m n}$ are the coefficients of Lagrange deformation tensor, $c_{i j k l}^{T}$ is isothermal first-order elastic coefficients, $F\left(\eta_{i j}, T\right)$ is the Helmholtz free energy.

The components of the stress tensor can be extracted by $\sigma_{i}=\sum_{j=1}^{6} c_{i j} \varepsilon_{j}$, after the applied strain, the total energy variation of the system can be expressed as:

$$
\Delta E=\frac{V}{2} \sum_{i=1}^{6} \sum_{j=1}^{6} c_{i j} e_{i} e_{j}
$$

As the independent elastic constants of different crystals are different from each other, through the different matrix elements of the crystal elastic modulus, we can get different strain applied methods. So, the second order elastic coefficients can be obtained by the coefficient of the second-order Taylor expansion of Helmholtz free energy with the strain,

$$
c_{i j k l}^{T}=\rho_{0} \frac{\partial^{2} F\left(\eta_{i j}, T\right)}{\partial \eta_{i j} \partial \eta_{k l}}
$$

Here, strain and thermodynamics deformation is symmetric. There is only six independent deformation tensor in the 9-dimensional deformation tensor. By DFT method, the electron density is optimised and elastic constants are obtained.

For monoclinic crystal structure, elastic constants include: $\mathrm{C}_{11}, \mathrm{C}_{22}, \mathrm{C}_{33}, \mathrm{C}_{12}, \mathrm{C}_{13}, \mathrm{C}_{23}, \mathrm{C}_{44}, \mathrm{C}_{55}$, $\mathrm{C}_{66} \mathrm{C}_{15}, \mathrm{C}_{25}, \mathrm{C}_{35}$ and $\mathrm{C}_{46}$. The criteria for mechanical stability are given by $\mathrm{Wu}^{[16]}$ :

$$
\begin{aligned}
& c_{i j}>0 \quad(i=1,2,3,4,5,6) \\
& \left(c_{44} c_{66}-c_{46}^{2}\right)>0 \\
& \left(c_{33} c_{55}-c_{35}^{2}\right)>0 \\
& \left(c_{22}+c_{33}-2 c_{23}\right)>0 \\
& {\left[c_{11}+c_{22}+c_{33}+2\left(c_{12}+c_{13}+c_{23}\right)\right]>0} \\
& {\left[c_{22}\left(c_{33} c_{55}-c_{35}^{2}\right)+2 c_{23} c_{25} c_{35}-c_{23}^{2} c_{55}-c_{25}^{2} c_{33}\right]>0} \\
& \left\{2\left[c_{15} c_{25}\left(c_{33} c_{12}-c_{13} c_{23}\right)+c_{15} c_{35}\left(c_{22} c_{13}-c_{12} c_{23}\right)+c_{25} c_{35}\left(c_{11} c_{23}-c_{12} c_{13}\right)\right]\right. \\
& \left.\quad-\left[c_{15}^{2}\left(c_{22} c_{33}-c_{23}^{2}\right)+c_{25}^{2}\left(c_{11} c_{33}-c_{13}^{2}\right)+c_{35}^{2}\left(c_{11} c_{22}-c_{12}^{2}\right)\right]+g c_{55}\right\}>0
\end{aligned}
$$


The homogenized elastic properties of polycrystals can be separately calculated, of which shear modulus and bulk modulus are as follows ${ }^{[16]}$ :

$$
\begin{aligned}
G_{V}= & \frac{1}{15}\left[c_{11}+c_{22}+c_{33}+3\left(c_{44}+c_{55}+c_{66}\right)-\left(c_{12}+c_{13}+c_{23}\right)\right] \\
G_{R}=15 & \left\{\left[\left(c_{33} c_{55}-c_{35}^{2}\right)\left(c_{11}+c_{22}+c_{12}\right)+\left(c_{25} c_{55}-c_{25} c_{55}\right)\left(c_{11}-c_{12}-c_{23}\right)+\left(c_{13} c_{35}-c_{15} c_{33}\right)\left(c_{15}+c_{25}\right)\right.\right. \\
+ & \left.\left.\left(c_{13} c_{55}-c_{15} c_{35}\right) \cdot\left(c_{22}-c_{12}-c_{23}-c_{13}\right)+\left(c_{13} c_{25}-c_{15} c_{23}\right)\left(c_{15}-c_{25}\right)+f\right] / \Omega+3\left[g / \Omega+\left(c_{41}+c_{66}\right) /\left(c_{41} c_{66}-c_{46}^{2}\right)\right]\right]^{-1} \\
B_{V}= & {\left[c_{11}+c_{22}+c_{33}+2\left(c_{12}+c_{13}+c_{23}\right)\right] / 9 } \\
B_{R}=\Omega & {\left[\left(c_{33} c_{55}-c_{35}^{2}\right)\left(c_{11}+c_{22}-2 c_{12}\right)+\left(c_{23} c_{55}-c_{25} c_{35}\right)\left(2 c_{12}-2 c_{11}-c_{23}\right)+\left(c_{13} c_{35}-c_{15} c_{33}\right) .\right.} \\
& \left.\left(c_{15}-2 c_{25}\right)+\left(c_{13} c_{55}-c_{15} c_{35}\right)\left(2 c_{12}+2 c_{23}-c_{13}-2 c_{22}\right)+2\left(c_{13} c_{25}-c_{15} c_{23}\right)\left(c_{25}-c_{15}\right)+f\right]^{-1} \\
f= & c_{11}\left(c_{22} c_{55}-c_{25}^{2}\right)-c_{12}\left(c_{12} c_{55}-c_{15} c_{25}\right)+c_{15}\left(c_{12} c_{25}-c_{15} c_{22}\right)+c_{25}\left(c_{23} c_{35}-c_{25} c_{33}\right) \\
g= & c_{11} c_{22} c_{33}-c_{11} c_{23}^{2}-c_{22} c_{13}^{2}-c_{33} c_{12}^{2}+2 c_{12} c_{13} c_{23} \\
\Omega=2 & {\left[c_{15} c_{25}\left(c_{33} c_{12}-c_{13} c_{23}\right)+c_{15} c_{35}\left(c_{22} c_{13}-c_{12} c_{23}\right)+c_{25} c_{35}\left(c_{11} c_{23}-c_{12} c_{13}\right)\right] } \\
& -\left[c_{15}^{2}\left(c_{22} c_{33}-c_{23}^{2}\right)+c_{25}^{2}\left(c_{11} c_{33}-c_{13}^{2}\right)+c_{35}^{2}\left(c_{11} c_{22}-c_{12}^{2}\right)\right]+g c_{55}
\end{aligned}
$$

In terms of the Voigt-Reuss-Hill approximations ${ }^{[17]}, M_{H}=(1 / 2)\left(M_{R}+M_{V}\right), M$ refers to $B$ or $G$. Based on the Voigt-Reuss-Hill approximation, the shear modulus, bulk modulus as well as Young's modulus $E$ and Poisson's ratio $\mu$ can be written as ${ }^{[18]}$.

$$
\begin{aligned}
& E=\frac{9 B G}{3 B+G}=\frac{9\left(B_{V} / 2+B_{R} / 2\right)\left(G_{V} / 2+G_{R} / 2\right)}{3\left(B_{V} / 2+B_{R} / 2\right)+\left(G_{V} / 2+G_{R} / 2\right)} \\
& \mu=\frac{3 B-2 G}{2(3 B+G)}=\frac{3\left(B_{V} / 2+B_{R} / 2\right)-2\left(G_{V} / 2+G_{R} / 2\right)}{6\left(B_{V} / 2+B_{R} / 2\right)+2\left(G_{V} / 2+G_{R} / 2\right)}
\end{aligned}
$$

Then homogenized elastic properties of polycrystals can be calculated, of which shear, bulk and Possion's ratio can be obtained by calculating Voigt and Reuss bounds and averaging term ${ }^{[16]}$. Then the Voigt-Reuss-Hill average ${ }^{[16]}$ will be determined and Young's modulus can be calculated from shear/bulk modulus.

\section{Modeling and homogenized elastic moduli of gypsum structure}

\section{Nanoscale modeling of monoclinic gypsum crystal}

The gypsum morphology is monoclinic and the initial lattice is as: $a=5.677 \AA, b=15.207 \AA$, $c=6.528 \AA, \alpha=\beta=90^{\circ}, \gamma=118.49^{\circ}$, its structure is monoclinic with space group I $2 / \mathrm{a}^{[19]}$.

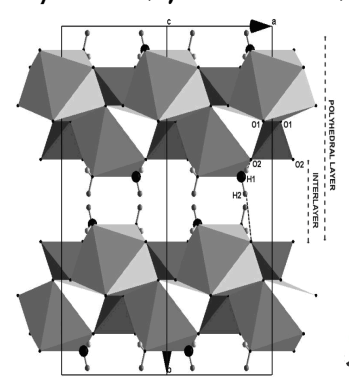

a) Gypsum structure ${ }^{[21]}$ along [001] b) The real cell
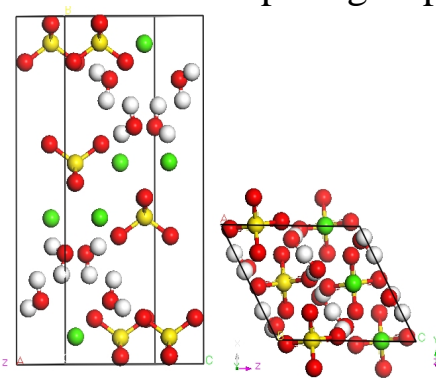

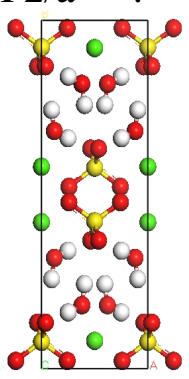

e)In $z$-direction

Fig. 1 Modeling of gypsum crystal.

In Fig.1, the gypsum crystal can be summarized as:

1). the two hydrogen atoms of water molecules formed weak hydrogen bonds with the $O$ atoms of $C a$ and $S$ polyhedra;

2). a stacking sequence of $\mathrm{CaO}_{8}$ and $\mathrm{SO}_{4}$ chains in the (010) plane alternate with water layers along the $b$-axis; 
3). in (010) plane, the sulfate tetrahedra and $\mathrm{CaO}_{8}$ polyhedra alternate to form edge sharing chains along [100] and zigzag chains along [001] direction ${ }^{[20]}$.

Table 1 Atomic coordinates and displacement parameters of gypsum ${ }^{[20]}$

\begin{tabular}{llllcc}
\hline atom & $\mathrm{x}$ & $\mathrm{y}$ & $\mathrm{z}$ & occupancy rate & Uiso or Ueq \\
\hline $\mathrm{Ca}$ & 0.5000 & 0.0786 & 0.2500 & 1.00 & 1.00 \\
$\mathrm{~S}$ & 0.0000 & 0.0787 & 0.7500 & 1.00 & 1.00 \\
$\mathrm{O} 1$ & -0.0384 & 0.1326 & 0.5512 & 1.00 & 1.00 \\
$\mathrm{O} 2$ & 0.2429 & 0.0215 & 0.8347 & 1.00 & 1.00 \\
$\mathrm{Ow}$ & 0.3784 & 0.1825 & 0.4554 & 1.00 & 1.00 \\
$\mathrm{H} 1$ & 0.2504 & 0.1615 & 0.5009 & 1.00 & 1.00 \\
$\mathrm{H} 2$ & 0.4022 & 0.2435 & 0.4900 & 1.00 & 1.00 \\
\hline
\end{tabular}

In order to determine whether the elastic coefficients of gypsum crystal under various pressures are stable, the pressure region of $0 \sim 1.0 G P a$ using DFT methods is added.

\section{Initial conditions and elastic constants of gypsum}

The initial conditions are as: the pressure region of $0 \sim 1 G P a$ is used. Besides, a plane-wave basis set, ultrasoft pseudopotentials using GGA are used with a plane-wave cutoff energy of $400 \mathrm{eV}$. Brillouin zone is $6 \times 6 \times 4$. Self-consistent convergence of the total energy per atom is chosen $10^{-4} \mathrm{eV}$. Elastic constants of gypsum model based on DFT are calculated (Table 2), and then elastic modulus can be obtained ${ }^{[16]}$.

Table 2 Elastic coefficient $C_{i j}(G P a)$ of gypsum by DFT.

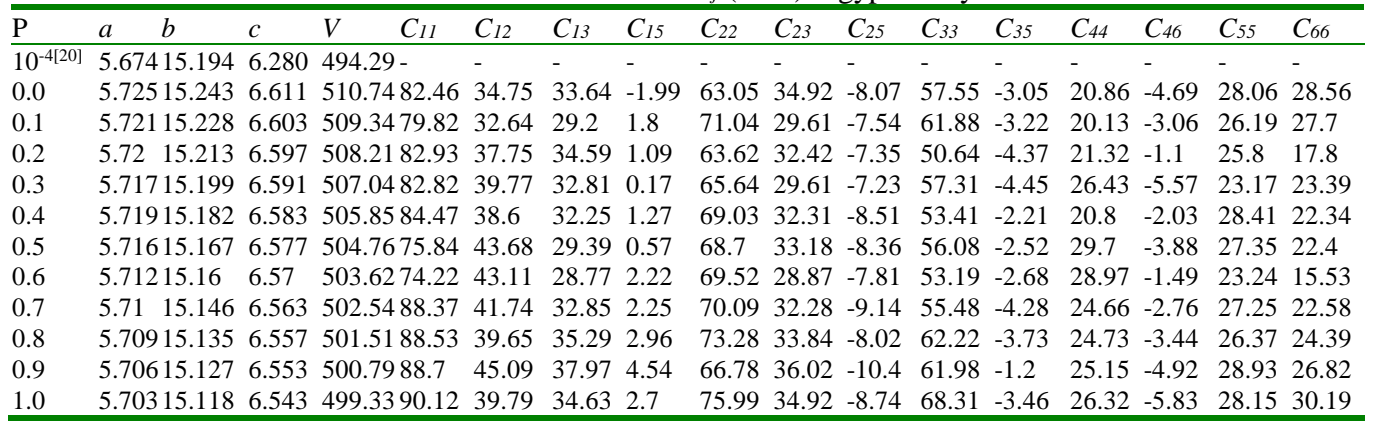

Elastic constants of monoclinic gypsum crystal under $0 \sim 1.0 \mathrm{GPa}$ are shown in Fig.2.

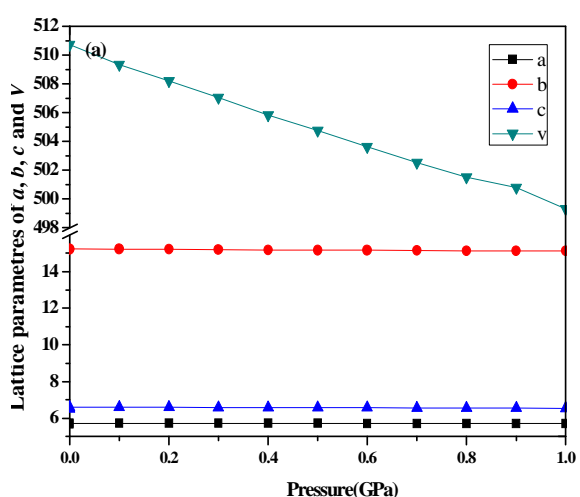

a) Relative change of $a, b, c$ and $V$

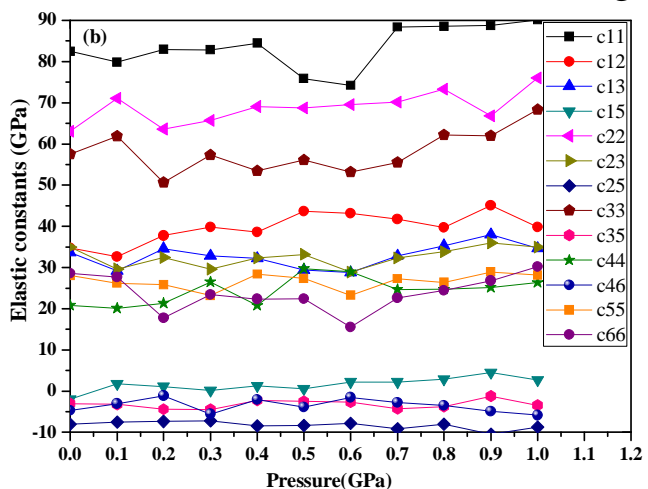

b) Elastic constants

Fig. 2 Gypsum monoclinic crystal under pressure $0 \sim 1.0 \mathrm{GPa}$ by DFT.

From Fig.2, elastic constants of gypsum crystal at $0 G P a$ are given as: $\mathrm{c}_{11}=82.464 G P a$, $\mathrm{c}_{12}=34.751 \mathrm{GPa}, \mathrm{c}_{13}=33.643 \mathrm{GPa}, \mathrm{c}_{15}=-1.987 \mathrm{GPa}, \mathrm{c}_{22}=63.046 \mathrm{GPa}, \mathrm{c}_{23}=34.920 \mathrm{GPa}, \mathrm{c}_{25}=-8.071 \mathrm{GPa}$, $\mathrm{c}_{33}=57.549 \mathrm{GPa}, \mathrm{c}_{35}=-3.054 G P a, \mathrm{c}_{44}=20.863 \mathrm{GPa}, \mathrm{c}_{46}=-4.688 \mathrm{GPa}, \mathrm{c}_{55}=28.062 \mathrm{GPa}, \mathrm{c}_{66}=28.556 \mathrm{GPa}$. It is found that the oxygen atom of the water molecule did not change its position or occupancy under pressure conditions. It agrees with the conclusion that a simple pressure increase at ambient temperature cannot induce dehydration because of the unchange of water molecular in the gypsum structure within pressure range ${ }^{[20]}$.

\section{Elastic modulus of monoclinic gypsum structure}

Based on elastic constants of gypsum crystal calculated by DFT, elastic moduli of gypsum structure at $0 \mathrm{GPa}$ are verified and averaged. DFT calculation results are in Fig.3. 
The measurement of these constants using an acoustic method has been investigated ${ }^{[21]}$. The elastic constants of gypsum crystal is given by reference ${ }^{[22]}$, thus elastic moduli by experiment are as: $G_{v}=26.533 \mathrm{GPa} ; B_{v}=39.256 \mathrm{GPa} ; G_{r}=24.808 \mathrm{GPa} ; B_{r}=39.238 \mathrm{GPa} ; E=63.227 \mathrm{GPa} ; \mu=0.2315$, and the corresponding values of moduli by reference ${ }^{[23]}: B=44 \mathrm{GPa}, G=17 \mathrm{GPa}, E=45.7 \mathrm{GPa}, \mu=0.33$.

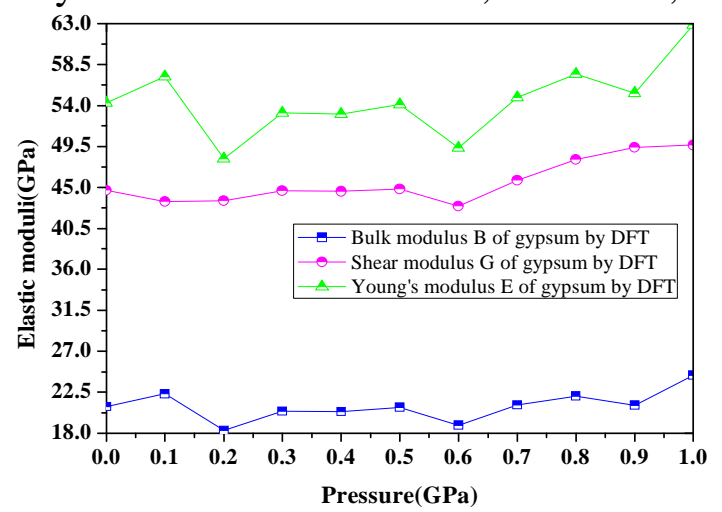

Fig. 3 Elastic moduli of gypsum crystal under pressure $0 \sim 1.0 \mathrm{GPa}$.

As gypsum shows anisotropic compressibility along three crystallographic axes with $b>c>a$ below $5 \mathrm{GPa}^{[24]}$, we take the pressure region of $0-1.0 \mathrm{GPa}$ to verify whether the performance of gypsum model under low pressure is stable. Mechanical moduli of gypsum polycrystalline by different methods are in Table 3.

Table 3 Mechanical moduli of gypsum polycrystalline by different methods

\begin{tabular}{|c|c|c|c|c|c|c|c|c|}
\hline Pressure (GPa) & $\mathrm{Gv}(\mathrm{GPa})$ & $\mathrm{Bv}(\mathrm{GPa})$ & $\mathrm{Gr}(\mathrm{GPa})$ & $\mathrm{Br}(\mathrm{GPa})$ & $\mathrm{B}(\mathrm{GPa})$ & $\mathrm{G}(\mathrm{GPa})$ & $\mathrm{E}(\mathrm{GPa})$ & $\mu$ \\
\hline Reference $^{[23]}$ & 26.5333 & 39.2556 & 24.8077 & 39.2381 & 25.6705 & 39.2469 & 63.2265 & 0.2315 \\
\hline 0.0 (GGA) & 22.1459 & 45.5208 & 19.7054 & 43.8224 & 20.9257 & 44.6716 & 54.2985 & 0.2974 \\
\hline 0.1 (GGA) & 22.8896 & 43.9624 & 21.7569 & 42.9250 & 22.3233 & 43.4437 & 57.1766 & 0.2806 \\
\hline 0.2 (GGA) & 19.1472 & 45.1906 & 17.4395 & 41.9064 & 18.2934 & 43.5485 & 48.1394 & 0.3158 \\
\hline 0.3 (GGA) & 21.5041 & 45.5718 & 19.3501 & 43.6675 & 20.4271 & 44.6197 & 53.1678 & 0.3014 \\
\hline 0.4 (GGA) & 21.2263 & 45.9146 & 19.5324 & 43.2463 & 20.3794 & 44.5805 & 53.0537 & 0.3017 \\
\hline 0.5 (GGA) & 22.1809 & 45.9026 & 19.4980 & 43.7566 & 20.8395 & 44.8296 & 54.1306 & 0.2988 \\
\hline 0.6 (GGA) & 19.9615 & 44.2709 & 17.7554 & 41.5818 & 18.8585 & 42.9264 & 49.3486 & 0.3084 \\
\hline 0.7 (GGA) & 22.0356 & 47.5189 & 20.1833 & 44.0623 & 21.1095 & 45.7906 & 54.8931 & 0.3002 \\
\hline 0.8 (GGA) & 22.7817 & 49.0659 & 21.3745 & 47.0681 & 22.0781 & 48.0670 & 57.4399 & 0.3008 \\
\hline 0.9 (GGA) & 22.7399 & 50.6242 & 19.4094 & 48.1542 & 21.0747 & 49.3892 & 55.3510 & 0.3132 \\
\hline $1.0(\mathrm{GGA})$ & 25.2707 & 50.3430 & 23.4785 & 48.9327 & 24.3746 & 49.6379 & 62.8382 & 0.2890 \\
\hline
\end{tabular}

An angular average, using equation (12) -(20) with the full elastic moduli tensor, leads to elastic moduli as: $G_{v}=22.146 \mathrm{GPa}, G_{r}=19.705 \mathrm{GPa}, B_{v}=45.521 \mathrm{GPa}, \quad B_{r}=43.822 \mathrm{GPa}, \quad B=44.672 \mathrm{GPa}$, $G=20.926 \mathrm{GPa}, E=54.299 \mathrm{GPa}, \mu=0.2974$. These results are close to the plane-strain value of Young's modulus by reference ${ }^{[24]} E=50 \mathrm{GPa}, \mu=0.45$.

As is known to us, if the moduli tensor is anisotropic, Young's modulus will scale with the different directions. By comparison of gypsum crystal and $\mathrm{CH}$ crystal, axial moduli of gypsum in $x, y$, $z$ directions are $57.75 \mathrm{GPa}, 37.22 \mathrm{GPa}$ and $34.91 \mathrm{GPa}$ while axial moduli of $\mathrm{Ca}(\mathrm{OH})_{2}$ in $x, y, z$ directions are $93.75 \mathrm{GPa}, 93.75 \mathrm{GPa}$ and $42.39 \mathrm{GPa}$, showing that gypsum crystal is much less anisotropic than hydrogen-bonded layered $\mathrm{Ca}(\mathrm{OH})_{2}$ structure ${ }^{[23]}$.

\section{Conclusions}

Elastic constants of gypsum crystal structures under a certain pressure region are calculated by DFT method, which has a certain value for both application and reference. Results are as:

(1) For monoclinic gypsum single crystal, elastic coefficients are obtained in 0 1 GPa pressure range to verify the reliability of the model by comparing other literatures.

(2) Elastic constants of gypsum single crystal at $0 \mathrm{GPa}$ are given as follows: $\mathrm{c}_{11}=82.464 \mathrm{GPa}$, $\mathrm{c}_{12}=34.751 \mathrm{GPa}, \mathrm{c}_{13}=33.643 \mathrm{GPa}, \mathrm{c}_{15}=-1.987 \mathrm{GPa}, \mathrm{c}_{22}=63.046 \mathrm{GPa}, \mathrm{c}_{23}=34.920 \mathrm{GPa}, \mathrm{c}_{25}=-8.071 \mathrm{GPa}$, $\mathrm{c}_{33}=57.549 \mathrm{GPa}, \mathrm{c}_{35}=-3.054 \mathrm{GPa}, \mathrm{c}_{44}=20.863 \mathrm{GPa}, \mathrm{c}_{46}=-4.688 \mathrm{GPa}, \mathrm{c}_{55}=28.062 \mathrm{GPa}, \mathrm{c}_{66}=28.556 \mathrm{GPa}$.

(3) Young's modulus of gypsum is about $54.299 \mathrm{GPa}$. Elastic moduli of gypsum at 0GPa are homogenized as: $G_{v}=22.146 \mathrm{GPa}, G_{r}=19.705 \mathrm{GPa}, B_{v}=45.521 \mathrm{GPa}, B_{r}=43.822 \mathrm{GPa}, B=44.672 \mathrm{GPa}$, $G=20.926 \mathrm{GPa}, E=54.299 \mathrm{GPa}, \mu=0.297$. 
Structural, elastic properties of typical crystals are investigated and Cij determination is given by DFT method. Reuss-Voigt-Hill estimation has been used for gypsum crystal structures and can be seen as an intermediate step in the homogenization of elastic properties.

\section{Acknowledgements}

The authors greatly acknowledge the financial support for this work provided by China Scholarship Council (CSC), Natural Science Foundation of China (No.21671096) and support of start-up foundation of Xi' an Shiyou University.

\section{References}

[1] B. Bary, C. Bourcier, T. Helfer: Advances in Engineering Software, Vol.112, (2017), p.16-30.

[2] S.D. Abyaneh, H.S. Wong, N.R. Buenfeld:Computational Materials Science, Vol.119, (2016), p.130-143.

[3] J. Liu, Z. Renbo, D. Xiuli: Construction and Building Materials, Vol.141, (2017), p.222-234

[4] I. S. Messaoudi, A.Zaoui, \& M. Ferhat: physica status solidi (b), Vol.252(3) (2015), p.490-495.

[5] Z. Qian, E. Schlangen, G. Ye, K. van Breugel: Materials, Vol.10 (6), (2017), p.587.

[6] J. Fu, F. Bernard, S. Kamali-Bernard: Journal of Nano Research. Vol.33,(2015), p.92-105.

[7] V. Nguyen, M. Stroeven, L.J. Sluys: Journal of multiscale modeling, Vol.3(04), (2011), p.229-270.

[8] J. Fu, F. Bernard, S. Kamali-Bernard: Molecular Simulation, Vol.43(20), (2017), p.1-15.

[9] T. Gnäupel-Herold: Powder Diffraction, Vol. 27(02), (2012), p.114-116.

[10] P. Wriggers, S.O. Moftah: Finite elements in analysis and design, Vol.42 (7), (2006), p.623-636.

[1 1] A. Reuss: Journal of Applied Mathematics and Mechanics/Zeitschrift für Angewandte Mathematik und Mechanik, Vol. 9 (1), (1929), p.49-58.

[12] W. Voigt: Leipzig, Teubner, 1928, New York, Johnson Reprint.

[13] H. Behnken, V. Hauk: Zeitschrift für Metallkunde, Vol. 77, (1986), p.620-626.

[14] J. Fu, F. Bernard, S. Kamali-Bernard: Journal of Physics and Chemistry of Solids, Vol.101, (2017), p.74-89.

[15] S. Kamali-Bernard, F. Bernard : Computational Materials Science, Vol. 47, (2009), p.178-185.

[16] Z.J. Wu, E.J. Zhao, H. P. Xiang, et al.: Physical Review B, Vol.76(5), (2007), p.054115.

[17] D.Raabe, Computational Materials Science: The Simulation of Materials Microstructures and Properties, Wiley-VCH, Weinheim, 1998.

[18] R. Hill: Proceedings of the Physical Society. Section A, Vol.65(5), (1952), p.349.

[19] K.S. Knight, I.C. Stretton, P.F. Schofield: Physics and chemistry of minerals, Vol.26(6), (1999), p.477-483.

[20] P. Comodi, S. Nazzareni, P.F. Zanazzi, S. Speziale: American Mineralogist, Vol.93(10), (2008), p.1530-1537.

[21] S. Haussuhl: Zeitschrift fur Kristallographie, Vol.122, 1960, p.311-314.

[22] S. Meille, E.J. Garboczi: Modelling and Simulation in Materials Science and Engineering, Vol.9(5), 2001, p.371.

[23] J.P. Watt: Journal of Applied Physics, Vol.51, (1980), p.1520-1524.

[24] E. Huang, J. Ku, J. Lin, J. Hu: High Pressure Research, Vol.17, (2000), p.57-75. 